\title{
A Cartesian Bicategory of Polynomial Functors in Homotopy Type Theory
}

\author{
Eric Finster \\ University of Cambridge
}

\author{
Samuel Mimram \\ École polytechnique
}

\author{
Maxime Lucas \\ Université Sorbonne Paris Nord
}

\author{
Thomas Seiller \\ CNRS*
}

\begin{abstract}
Polynomial functors are a categorical generalization of the usual notion of polynomial, which has found many applications in higher categories and type theory: those are generated by polynomials consisting a set of monomials built from sets of variables. They can be organized into a cartesian bicategory, which unfortunately fails to be closed for essentially two reasons, which we address here by suitably modifying the model. Firstly, a naive closure is too large to be well-defined, which can be overcome by restricting to polynomials which are finitary. Secondly, the resulting putative closure fails to properly take the 2-categorical structure in account. We advocate here that this can be addressed by considering polynomials in groupoids, instead of sets. For those, the constructions involved into composition have to be performed up to homotopy, which is conveniently handled in the setting of homotopy type theory: we use it here to formally perform the constructions required to build our cartesian bicategory, in Agda. Notably, this requires us introducing an axiomatization in a small universe of the type of finite types, as an appropriate higher inductive type of natural numbers and bijections.
\end{abstract}

\section{Introduction}

Polynomial functors have been introduced as a categorical generalization of traditional polynomials and have been intensively studied by Kock and collaborators $[9,16,10]$. They have become an important categorical tool, allowing the definition and manipulation of various structures such as opetopes [17] or type theories [3]. This motivates the study of the categorical structures they bear in order to facilitate constructions on those, and the situation turns out to be quite subtle. For instance, one would expect that they should be cartesian closed (after all, most usual categories are), but it is not the case: the cartesian product functor does not have a satisfactory right adjoint. There are essentially two reasons for that.

The first one is that there are size issues: the naive definition of the exponential appears to be too large to be a proper object in the category of polynomials. This is easily overcome by restricting to polynomials which are finitary, i.e., only involve monomials consisting of products of variables which are finite. The resulting category is isomorphic to the category of Girard's normal functors [11], which is a model of simply typed $\lambda$-calculus, and linear logic [12], which can be thought of as a quantitative variant of the relational model (historically, this model is in fact one of the starting points motivating the introduction of linear logic).

The second one is more problematic: polynomial functors carry an intuitive 2-categorical structure, but it was observed early on that the closure mentioned above fails to extend to a 2-categorical one [11, $20,12]$. Here, we advocate that a satisfactory answer to this problem is provided by switching from traditional polynomial functors to ones over groupoids, as first considered by Kock [16], see also [23, 22]. We show that the resulting bicategory is cartesian closed; it is more generally a model of intuitionistic linear logic, which we expect to extend as a model of differential linear logic. The resulting category is

* Supported by the Île-de-France region through the DIM RFSI project CoHOp.

Ana Sokolova (Eds.): MFPS 2021

EPTCS 351, 2021, pp. 67-83, doi:10.4204/EPTCS.351.5
(C) E. Finster, S. Mimram, M. Lucas, T. Seiller

This work is licensed under the

Creative Commons Attribution License. 
close to the "equivariant variant" of polynomials, provided by generalized species or analytic functors [5, $8,7,6]$.

In order to assist us in our proofs and help us gain confidence in those, we have formalized most of them, from the beginning, in Agda, in the setting of univalent type theory [21] (with a custom implementation of homotopy type theory). Since this reflects the way we worked, allows easily manipulating objects such as groupoids as 1-truncated types, and ensures for free the functoriality and homotopy invariance of all constructions, we decided to present our results directly in the type-theoretic formalism, and a "translation" into the traditional set-theoretic setting for polynomial functors is planned for future works. Moreover, the present work required us to develop specific type-theoretic constructions, which we think could find application outside the scope of this work. A salient contribution is the construction, as a higher inductive type, of the type of finite sets and bijections in a small universe. The Agda code is publicly available [4].

We recall the traditional definition of polynomials, polynomial functors and associated constructions in section 2, we then present a formalization of the bicategory of polynomial functors in groupoids in section 3 and discuss the cartesian structure and the failure of being properly closed in section 4 . We then introduce and study the notion of finite type in section 5 and finally properly construct a cartesian closed bicategory in section 6 .

\section{Polynomial functors}

We begin by recalling the traditional definition of polynomial functors, as well as related constructions. All the material in this section is already known, but required in the following.

\subsection{The category of polynomial functors}

We briefly recall here the categorical generalization of the notion of polynomial provided by polynomial functors, and refer the reader to $[9,15]$ for a detailed presentation. Polynomials as traditionally defined as finite sums of the form $P(X)=\sum_{0 \leq i<k} X^{n_{i}}$. This notion can be "categorified" by taking a set $B$ of monomials (instead of specifying their number $k$ ) and having a set $E_{b}$ of instances of $X$ in each monomial $b$ (instead of specifying their number $n_{i}$ ). This data can thus collected as a function $P: E \rightarrow B$ representing the polynomial, where $B$ is the set of monomials and for each monomial $b, E_{b}=P^{-1}(b)$ is the set of instances of $X$ involved in the monomial. Such a function $P$, induces a functor $\llbracket P \rrbracket:$ Set $\rightarrow$ Set defined, by mimicking the usual definition of polynomials, as

$$
\llbracket P \rrbracket(X)=\sum_{b \in B} X^{E_{b}}
$$

and we call polynomial functor such a functor. An interesting point of view on the above data consists in considering the elements of $B$ as abstract operations, whose parameters are the elements of $E_{b}$, so that $\llbracket P \rrbracket(X)$ corresponds to the set obtained by formally applying the operations in $B$ to the required number of elements of the set $X$.

We will more generally consider the "typed" or "colored" variant of polynomials and polynomial functors, where the parameters of an operation are decorated by a "color" in a set $I$, as well as their output in a set $J$. This data can be encoded by a diagram $P$ in Set of the form

$$
I \stackrel{s}{\longleftarrow} E \stackrel{p}{\longrightarrow} B \stackrel{t}{\longrightarrow} J
$$


which we call a polynomial and consists of an uncolored polynomial $p$ together with functions $s$ and $t$ respectively indicating the colors of the parameters and outputs of operations. Note that the previous uncolored setting is recovered when $I$ and $J$ are both the terminal set. Writing Set/ $I$ for the slice category of sets over a set $I$, such data again induces a functor $\llbracket P \rrbracket:$ Set $/ I \rightarrow$ Set $/ J$, called a polynomial functor, obtained as the composite $\llbracket P \rrbracket=\Sigma_{t} \circ \Pi_{p} \circ \Delta_{s}$ where $\Delta_{s}$ is the pullback map along $s$, and $\Sigma_{t}$ (resp. $\Pi_{p}$ ) is the left (resp. right) adjoint to $\Delta_{t}$ (resp. $\Delta_{p}$ ) given by post-composition by $t$ (resp. local cartesian closure).

Alternatively, polynomial functors can be considered as acting on families instead of slice categories. Given a set $I$, it is well known that the slice category Set/I of sets over $I$ is equivalent to the category of families indexed by $I$,

$$
\text { Set } / I \simeq \text { Set }^{I}
$$

and, through this equivalence, the associated polynomial functor is $\llbracket P \rrbracket: \mathbf{S e t}^{I} \rightarrow \mathbf{S e t}^{J}$ such that

$$
\llbracket P \rrbracket\left(X_{i} \mid i \in I\right)=\left(\sum_{b \in t^{-1}(j)} \prod_{e \in p^{-1}(b)} X_{s(e)} \mid j \in J\right)
$$

It can be shown (and this is non-trivial) that the composite of two polynomial functors is again polynomial: this means that given two polynomial functors generated by two diagrams of the form (1), one can find a third diagram of the form (1) which is a presentation for the composite of the functors. We can thus build a category PolyFun where an object is a set and a morphism $I \rightarrow J$ is a polynomial functor Set $/ I \rightarrow \operatorname{Set} / J\left(\right.$ or $\operatorname{Set}^{I} \rightarrow \mathbf{S e t}^{J}$ ). Note that even though an operation of composition is defined on polynomials (1), we cannot build a category of those: their composition being defined by using universal constructions, it will not be strictly associative and the best we can hope for is a structure of a bicategory. This motivates investigating the 2-categorical structure of polynomials.

\subsection{2-categorical structure}

Given two polynomials $P$ and $P^{\prime}$ of the form (1), both from $I$ to $J$, a morphism between them consists of two functions $\beta: B \rightarrow B^{\prime}$ and $\varepsilon: E \rightarrow E^{\prime}$ between the operations (resp. parameters) of $P$ and those of $P^{\prime}$ making the diagram

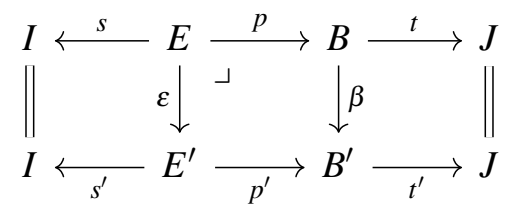

commute and such that the middle square is a pullback (such a morphism is sometimes said to be cartesian to insist on this requirement). This last condition can be understood as requiring that $\beta$ preserves the arity of operations (it is also technically important because there is no sensible way of defining horizontal composition of morphisms without this condition). The composition of polynomials defined above turns out to be associative up to isomorphism, so that one can define a bicategory Poly whose 0-cells are sets, 1-cells are polynomials and 2-cells are morphisms of polynomials.

The resulting bicategory can be shown to be biequivalent to the 2-category PolyFun, obtained by adding suitable natural transformations as 2-cells to the above category [9, Theorem 2.17]. There is a subtlety concerning the 2-cells: the "natural" notion of morphism between polynomial functors, strong natural transformations, is more liberal than the notion of morphism defined above on corresponding polynomials, and one can either restrict those transformations (to cartesian natural transformations) or generalize the notion of morphism between polynomials. 


\subsection{Cartesian closed structure}

The 1-category PolyFun of polynomial functors is cartesian. Considering polynomial functors as operating on families through (2), as explained above, the paring of two polynomial functors $P: I \rightarrow J$ and $Q: I \rightarrow K$ is induced by the one in Cat:

$$
\langle P, Q\rangle: \operatorname{Set}^{I} \rightarrow \operatorname{Set}^{J} \times \operatorname{Set}^{K} \cong \operatorname{Set}^{J \sqcup K}
$$

which motivates defining the product on objects of PolyFun as the coproduct of sets. We can hope that the category also has a closure for products induced by the one in Cat. The sequence of bijections of hom-sets

suggests that we define the closure as

$$
\operatorname{Set}^{I} \times \operatorname{Set}^{J} \rightarrow \operatorname{Set}^{K} \cong \operatorname{Set}^{I} \rightarrow\left(\operatorname{Set}^{K}\right)^{\operatorname{Set}^{J}} \cong \operatorname{Set}^{I} \rightarrow \operatorname{Set}^{\operatorname{Set}^{J} \times K}
$$

$$
[J, K]=\operatorname{Set}^{J} \times K \simeq \operatorname{Set} / J \times K
$$

However, this does not make sense because the objects of PolyFun are sets and this lives in a larger universe. This motivates considering polynomials which are "reasonably small". We say that a polynomial $P$ of the form (1) is finitary when every operation has a finite set of parameters, i.e., the set $E_{b}=p^{-1}(b)$ is finite for every operation $b$. Polynomial functors corresponding to finitary functors can be shown to be those preserving filtered colimits and are sometimes called normal functors [11, 12]. From now on, we restrict our category to such polynomials, and consider them up to isomorphism. This change allows us to replace Set $/ J$ by $\mathbf{S e t}_{\text {fin }} / J$ in the above definition of the exponential, where $\mathbf{S e t}_{\text {fin }}$ is the class of finite sets (i.e., we consider finite families of set). While this is certainly "smaller", this is still not a proper set; however, it is now equivalent to a proper set, namely the set $\mathbb{N} / J$ of functions $[n] \rightarrow J$ for some $n \in \mathbb{N}$, where $[n]=\{0, \ldots, n-1\}$ is a canonical choice of a finite set with $n$ elements. To sum up, it can be shown that the resulting category is still cartesian and we can define the exponential as $[J, K]=\mathbb{N} / J \times K$.

We have seen that the category of polynomial functors really is a 2-category (or a bicategory if we consider polynomials instead) and it is natural to expect that the cartesian closure would extend to the 2-categorical setting, by which we mean that the isomorphism

$$
\operatorname{PolyFun}(I \sqcup J, K) \cong \operatorname{PolyFun}(I, \mathbb{N} / J \times K)
$$

between sets of isomorphism classes of polynomial functors should extend to an equivalence of categories. It has however been observed that it is not the case, see [11, Remark 2.19], [20, Example 1.4.2] and [12, Theorem 1.24]. As an illustration, consider the polynomial functor $\llbracket P \rrbracket(X)=X^{2}$, which is induced by the polynomial $P$ given by the diagram $1 \leftarrow 2 \rightarrow 1 \rightarrow 1$ (we write $n$ for a set with $n$ elements). It can be remarked that there are two automorphisms on the polynomial $P$ : the identity and the morphism of the form (3) where $\varepsilon: 2 \rightarrow 2$ is the transposition. The exponential transpose of $P: 0 \sqcup 1 \rightarrow 1$ is the polynomial $P^{\sharp}: 0 \rightarrow \mathbb{N} / 1 \times 1$ (whose target is isomorphic to $\mathbb{N}$ ) induced by the diagram $0 \leftarrow 0 \rightarrow 1 \rightarrow \mathbb{N}$, where the morphism $1 \rightarrow \mathbb{N}$ sends the element of 1 to $2 \in \mathbb{N}$ (which is the arity of the unique operation of $P$ ). Because 0 and 1 are respectively initial and terminal in sets, the identity is the only automorphism of $P^{\sharp}$, whereas the two automorphisms of $P$ should induce two automorphisms on its exponential transpose.

\subsection{Toward polynomial functors in groupoids}

This tension is solved in [12] by quotienting the 2-cells under an ad-hoc equivalence relation. In this paper, we advocate that a more satisfactory approach consists in switching from polynomial functors in 
sets to polynomial functors in groupoids: intuitively, the problem comes from the fact that, $\mathbb{N}$ being a set, there is no non-trivial endomorphism on a natural number, which we should have if we were to have a closure for the cartesian product. By switching to groupoids, we will be able to replace the set $\mathbb{N}$ in the above construction by the groupoid $\mathbf{B}$, which also has the natural numbers as objects, whose morphisms are all automorphisms, such that the group of automorphism on an object $n$ is the $n$-th symmetric group.

A proper definition of polynomials in groupoids requires more than simply considering diagrams of the form (1) in the category of groupoids. For instance, the traditional definition of composition does not immediately extend to those because the category of groupoids is not locally cartesian closed, and thus the right adjoint to the change of base functor $\Delta_{f}$ is not defined for every morphism $f$, which prevents us from making an immediate generalization of the definition of polynomial functors (a way to address this consists in restricting to polynomials (1) where $p$ and $t$ are fibrations [22]). Following [16], this can be explained as the fact that switching from sets to groupoids can be thought of as switching from 0 -truncated spaces to 1-truncated spaces, where the strict universal limits and colimits involved in the constructions on polynomials are not the right ones: we need to take limits and colimits up to homotopy. An important byproduct of working in homotopy type theory as we do is that all internal constructions are invariant up to homotopy, which avoids us explicitly dealing with those issues.

\section{The bicategory of polynomial functors}

We now present our formalization in homotopy type theory of the main steps for constructing the bicategory of polynomial functors. To be precise, we formalize here the sub-bicategory of the usual one, where we only keep invertible 2-cells (for which the problem for defining the closure is still non-trivial). The developments have been performed in Agda, and are available in the repository [4] based on our own formalization of homotopy type theory (or HoTT), following the reference book [21], to which we suppose the reader already acquainted.

We unfortunately do not have enough space here to expose in details the basic definitions of Agda and homotopy type theory, and only recall some notations. We write Type for the universe of small types and Type ${ }_{1}$ for the universe of large types (in particular Type is an element of Type ${ }_{1}$ ). We write $x \equiv y$ for the type of identities (or equalities or paths) between two terms $x$ and $y$ of the same type. We write $A \simeq B$ for the type of equivalences between two types $A$ and $B$ (possibly in different universes): it consists of functions $f: A \rightarrow B$ admitting an inverse up to homotopy, in a suitably coherent sense. We recall that a proposition is a type in any two elements are equal, and a set (resp. a groupoid) is a type in which the type $x \equiv y$ of identities between two elements $x$ and $y$ is a proposition (resp. a set). We postulate here the univalence axiom which states that the canonical map from identities $x \equiv y$ to equivalences $x \simeq y$ is itself an equivalence.

\subsection{Formalizing polynomials}

Our formalization of polynomials can be found in [4, Polynomial .agda]. The direct translation of the definition (1) of polynomials, as consisting of two types $E$ and $B$ and three functions $t, p$ and $s$ turns out to be quite cumbersome, because it involves quite a lot of manipulations of identities, even for the basic constructions of the category of polynomials. In the light of the equivalence (2), it is much more convenient to take the family point of view instead of the slice one, and use the following definition, also known in the literature as an indexed container [2]. Namely, in a polynomial (1), the function $t: B \rightarrow J$ associates to each operation of the polynomial in $B$ a color in $J$ : this data can equivalently be 
encoded as a family of types $0 p: J \rightarrow$ Type which to every element $j$ of $J$ associates the type $0 p \quad j$ of operations colored by $j$ of the polynomial. By performing similar transformations on the rest of the data, we reach the following definition, which is easier to work with because it uses more heavily dependent types: the explicit computations we had to perform with equality above are now implicitly handled by the dependent pattern matching of Agda.

Definition 1. The type of polynomials between two types I and $\mathrm{J}$ is

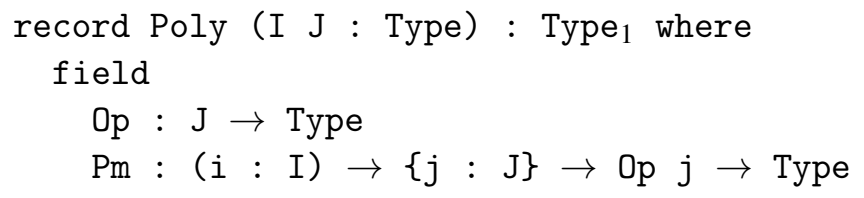

Given types I and $\mathrm{J}$, a polynomial from I to $\mathrm{J}$ consists of:

- a family of types Op $\mathrm{j}$ indexed by the elements $\mathrm{j}$ of $\mathrm{J}$ : the operations of the polynomial;

- a family of type Pm $i$ b indexed by the element $i$ of $I$ and the operations $b$ in Pm $j$ for some $j$ of $J$ (curly brackets indicate that this argument is usually left implicit): the parameters of the polynomial.

The careful reader will note that the above actually defines "polynomials in types", where there is a type (as opposed to a set or a groupoid) of operations and parameters. The notion of polynomial in groupoids can be obtained by further restricting to the case where all the involved types (I, J, Op j and Pm $i$ b) are groupoids, in the sense of HoTT recalled above.

\subsection{First constructions on polynomials}

The identity polynomial on a type $I$ is defined as

Id : Poly I I

Op Id $i=T$

$\operatorname{Pm} \operatorname{Id} i\{j=j\}$ tt $=i \equiv j$

and has, for each element $i$ of $I$, exactly one operation of type $i$, whose only parameter is also of type $i$. The polynomial functor induced by a polynomial $P$ from $I$ to $J$ is

$\llbracket-\rrbracket:$ Poly I J $\rightarrow$ ( $\rightarrow$ Type $) \rightarrow(\mathrm{J} \rightarrow$ Type $)$

$\llbracket \_\rrbracket P X j=\Sigma(O p P j)(\lambda c \rightarrow(i: I) \rightarrow(p: P m P i c) \rightarrow(X i))$

Finally, the composite $\mathrm{P} \cdot \mathrm{Q}$ of two polynomials $\mathrm{P}$ from $\mathrm{I}$ to $\mathrm{J}$, and $\mathrm{Q}$ from $\mathrm{J}$ to $\mathrm{K}$ is

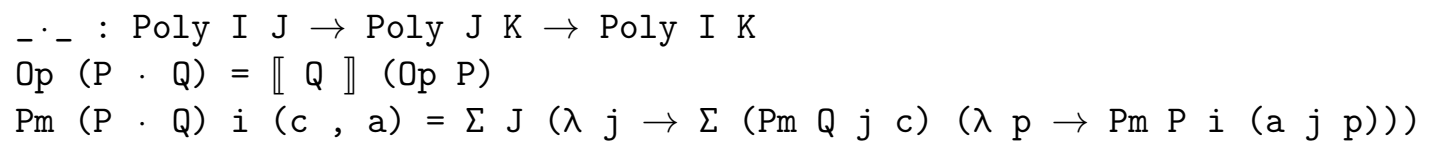

Morphisms between polynomials can be encoded as follows:

Definition 2. The type of morphisms between two polynomials $\mathrm{P}$ and $\mathrm{Q}$ is

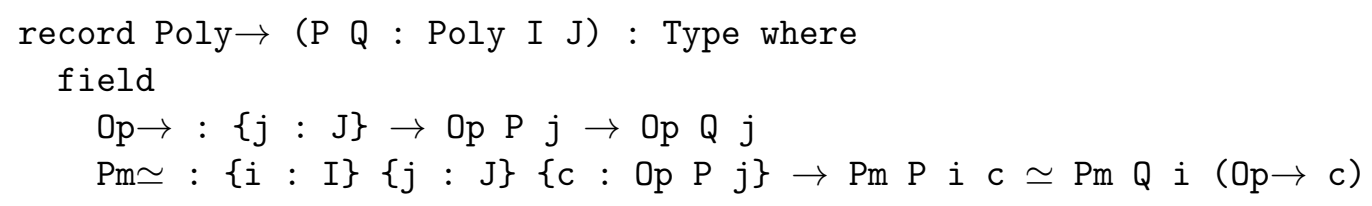

A morphism thus consists of: 
- a morphism between the operations of $\mathrm{P}$ and those of $\mathrm{Q}$, which respects the typing;

- a morphism between the parameters of $P$ and those of $Q$, which respects typing and operations, and is moreover an equivalence: this last requirement corresponds precisely to imposing that the square in the middle of (3) is a pullback.

We write I $\rightsquigarrow J$ (resp. P $\rightsquigarrow_{2}$ Q) for the type of polynomials from I to J (resp. morphisms of polynomials from $P$ to $Q)$.

Definition 3. A morphism $\varphi$ as above is an equivalence of polynomials when the morphism on operations is an equivalence at each element of $\mathrm{J}$ :

Poly-equiv : $\{P Q: I \rightsquigarrow J\}(\varphi: P \rightsquigarrow 2 Q) \rightarrow$ Type

Poly-equiv $\varphi=\{j: J\} \rightarrow$ is-equiv $(0 \mathrm{p} \rightarrow \varphi\{j=j\})$

We write $\mathrm{P} \simeq_{2} \mathrm{Q}$ for the type of equivalences of polynomials between $\mathrm{P}$ and $\mathrm{Q}$.

\subsection{A bicategory of polynomials}

Starting from there we can build all the structure one expects to find in a bicategory of polynomials:

- we can define the identity polynomial and the composition of polynomials (see above);

- we can show that composition of polynomials is associative and unital up to an equivalence of polynomials;

- we can define the horizontal and vertical composition of morphism of polynomials;

- we can show that those compositions are associative and unital up to a suitable notion of equivalence of morphisms of polynomials.

Moreover, by using univalence, one can show the following.

Proposition 4. The type of equivalences between two polynomials $\mathrm{P}$ and $\mathrm{Q}$ is equivalent to the type $\mathrm{P} \equiv \mathrm{Q}$ of equalities between the two polynomials: $(\mathrm{P} \equiv \mathrm{Q}) \simeq\left(\mathrm{P} \simeq_{2} \mathrm{Q}\right)$.

We can therefore build a bicategory (it might be more accurate to call it a $(2,1)$-category since 2-cells are equivalences) of groupoids, polynomials in groupoids and equivalences, in the following sense.

Definition 5. A prebicategory consists of:

- a type of ob objects;

- for each objects I and J, we have a groupoid hom I J of morphisms;

- for each object I there is a distinguished morphism id in hom I I called identity;

- there is a composition operation

_ $\otimes_{-}:$hom I J $\rightarrow$ hom J K $\rightarrow$ hom I K

for every objects I, J and K;

- composition is associative and unital:

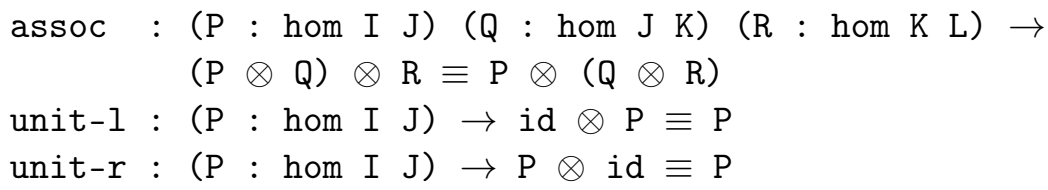


- the traditional pentagon law

$\operatorname{ap}(\lambda P \rightarrow P \otimes S)(\operatorname{assoc} P Q R) \cdot \operatorname{assoc} P(Q \otimes R) S \cdot$

$\operatorname{ap}(\lambda Q \rightarrow P \otimes Q)(\operatorname{assoc} Q R S) \equiv \operatorname{assoc}(P \otimes Q) R S \cdot \operatorname{assoc} P Q(R \otimes S)$

and triangle law

assoc $P$ id $Q \cdot \operatorname{ap}(\lambda Q \rightarrow P \otimes Q)($ unit-l $Q) \equiv$ ap $(\lambda P \rightarrow P \otimes Q)$ (unit-r $P$ )

of bicategories are satisfied for composable morphisms $P, Q, R$ and $S$.

Above, "." denotes the concatenation of paths (or transitivity of equality) and ap is a proof that every function is a congruence for equality.

Theorem 6. There is a prebicategory whose objects are groupoids, 1-cells are polynomials in groupoids and 2-cells are equivalences of polynomials.

The notion of prebicategory generalizes the notion of precategory in HoTT [21, Section 9.1]. As the name suggests, it lacks a property in order to bear the name of a bicategory, similarly to the situation with categories. A morphism $\mathrm{P}$ in hom $\mathrm{I} \mathrm{J}$ in a prebicategory is an internal equivalence when there exists morphisms $Q$ and $Q^{\prime}$ both in hom $J$ I such that $P \otimes Q \equiv i d$ and $Q^{\prime} \otimes P \equiv i d$, and we write $\mathrm{I} \simeq{ }^{\prime} \mathrm{J}$ for the type of internal equivalences from $\mathrm{I}$ to $\mathrm{J}$. There is a canonical map associating to any equality of type $\mathrm{I} \equiv \mathrm{J}$ between two objects I and $\mathrm{J}$ an internal equivalence from I to J. A bicategory is a prebicategory in which this canonical map is an equivalence, i.e., we have $(\mathrm{I} \equiv \mathrm{J}) \simeq(\mathrm{I} \simeq \mathrm{J})$ for every objects I and $\mathrm{J}$.

Theorem 7. The prebicategory of theorem 6 is a bicategory.

Note that proposition 4, in addition to proving the above theorem, allows us to use equalities as 2-cells instead of morphisms in the sense of definition 3. Because of this, the usual structure of bicategory which is "missing" from definition 5 (e.g. horizontal and vertical composition of 2-cells, the exchange law, etc.) is automatically present thanks to the general properties of equality. If this was not the case (for instance, if we wanted to consider morphisms of polynomials instead of equivalences as 2-cells), we would have had to use a much more involved notion of bicategory [1].

\section{Naive cartesian closed structure}

\subsection{Cartesian structure}

The notion of being cartesian for such a bicategory can be formalized in the expected way, by requiring the existence of a terminal object $\mathrm{T}$, a binary product operation $\oplus$ on objects and projection operations projl : hom $(I \oplus J) I$ and projr : hom $(I \oplus J)$ J for every objects I and $\mathrm{J}$, such that hom $\mathrm{I} \mathrm{T} \equiv \top$ and the canonical function hom I $(\mathrm{J} \oplus \mathrm{K}) \rightarrow$ hom I $\mathrm{J} \times$ hom I K (obtained by postcomposition with the projection operations) is an equivalence (and thus an equality by univalence). One can show:

Theorem 8. The bicategory of theorem 7 is cartesian, with the product being defined by coproduct $\sqcup$ on objects (the groupoids), first projection polynomial and pairing operation on polynomials being

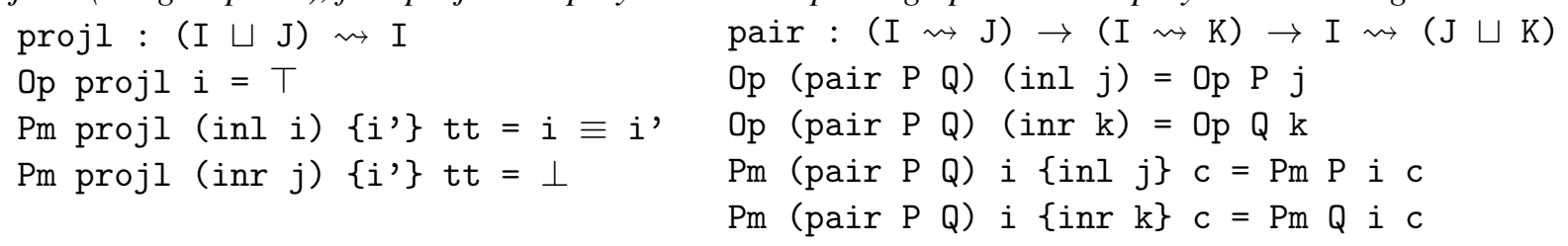

(and second projection is similar to first projection). 


\subsection{Naive closed structure}

A first step toward constructing a right adjoint to the product is the formalization of the naive closure described in section 2.3 given in [4, LargePolynomial.agda]. Namely, the formula (4) indicates that the hom space from I to $\mathrm{J}$ should be $(\mathrm{I} \rightarrow$ Type $) \times \mathrm{J}$. Of course, we encounter the same size issues as mentioned in the introduction, and we need to suppose that Type is the same as Type 1 (i.e., we disable the checking of universe levels), which makes the logic inconsistent, for this proof to go through. The formalization is still useful because it is a simple version of the actual one for the closure, which is more involved but does not require the extra assumption.

We define the exponential of a type as

Exp : Type $\rightarrow$ Type $_{1}$

$\operatorname{Exp} I=I \rightarrow$ Type

so that the internal hom between two types $I$ and $J$ should be Exp I $\times$ J. We can indeed define a currying map

curry : ( $\mathrm{I} \sqcup \mathrm{J}) \rightsquigarrow \mathrm{K} \rightarrow \mathrm{I} \rightsquigarrow(\operatorname{Exp} \mathrm{J} \times \mathrm{K})$

Op (curry P) $(j j, k)=\Sigma($ Op P k $)(\lambda c \rightarrow((\lambda j \rightarrow \operatorname{PmP}($ inr j $) c) \equiv j j))$

Pm (curry P) i $c=P m P$ (inl i) (fst c)

which formally transforms an operation with a given set of inputs in $\mathrm{J}$ into an operation with corresponding family of elements in $\mathrm{J}$ as output (the inputs in I are preserved and the output in $\mathrm{K}$ is preserved as the second component of the output). This could be graphically pictured as
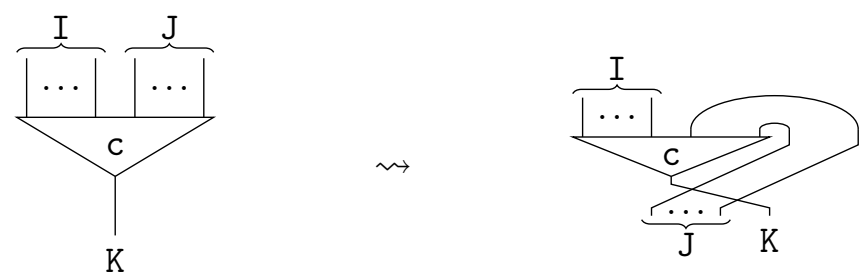

Similarly, one can also define an uncurrying map

uncurry : $\mathrm{I} \rightsquigarrow(\operatorname{Exp} \mathrm{J} \times \mathrm{K}) \rightarrow(\mathrm{I} \sqcup \mathrm{J}) \rightsquigarrow \mathrm{K}$

Op (uncurry P) $k=\Sigma(\operatorname{Exp} J)(\lambda j j \rightarrow O p P(j j, k))$

Pm (uncurry P) (inl $i)(j j, c)=P m P i c$

Pm (uncurry P) (inr $j)(j j, c)=j j j$

Theorem 9. The above maps induce an adjunction: we have $((\mathrm{I} \sqcup \mathrm{J}) \rightsquigarrow \mathrm{K}) \equiv(\mathrm{I} \rightsquigarrow(\operatorname{Exp} \mathrm{J} \times \mathrm{K}))$.

Proof. We can show that the two above maps are mutually inverse in the sense that we have

(uncurry (curry $\left.\mathrm{P}) \simeq_{2} \mathrm{P}\right)$

and

(curry (uncurry $\left.\mathrm{P}) \simeq_{2} \mathrm{P}\right)$

By proposition 4 , the equivalences of polynomials $\simeq_{2}$ can be turned into equalities: curry and uncurry thus form an equivalence, and we deduce the required equality by univalence.

An alternative definition for the "large exponential" can be given as follows. The equivalence (2) between slices and families generalizes in type theory. Given a type I, we have an equivalence (and thus an equality by univalence) between types over I and families of types indexed by I, see [4, Fam. agda]: 
Theorem 10. Given a type $\mathrm{I}$, we have the equivalence $(\mathrm{I} \rightarrow$ Type $) \simeq(\Sigma$ Type $(\lambda \mathrm{A} \rightarrow \mathrm{A} \rightarrow \mathrm{I}))$. This indicates that we could equivalently have taken the right member of the above equivalence as a definition of Exp I above.

\section{Finite types}

Following the plan of section 2.3 for the proof, we will restrict to finitary polynomials in order to have a smaller exponential in the closure, for which we can handle the size issues. In this section, we first formalize the notion of finiteness for a type, which will then be used to define finitary functors.

\subsection{Definition and properties}

The proofs associated to this section can be found in [4, FinType.agda]. As customary, we write Fin $\mathrm{n}$ for the canonical type with $n$ elements, its constructors being the natural numbers 0 up to $n-1$. Given a type A, we write $\|$ A $\|$ for its propositional truncation: an element of this type can be thought of as a witness that there exists a proof of A, without explicitly providing such a proof [21, Section 3.7].

Definition 11. A type is finite when it is merely equivalent to the type with $n$ elements for some natural number $n$ :

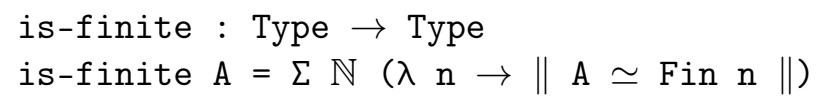

Remark 12. In case one wonders why we chose to use a truncation in the above definition, let us mention that using the definition is-finite' $A=\Sigma \mathbb{N}(\lambda \mathrm{n} \rightarrow \mathrm{A} \simeq$ Fin $\mathrm{n}$ ) would be bad because is-finite' $A$ is not a proposition, is-finite' $A$ is a large type, and the collection of all finite types is actually the natural numbers in the sense that one can construct an equivalence $\Sigma$ Type is-finite' $\simeq \mathbb{N}$.

The following lemma 14 shows that, for a finite type A, there is a well-defined notion of cardinality which is the natural number $\mathrm{n}$ such that $\| \mathrm{A} \simeq$ Fin $\mathrm{n} \|$ holds. In order to show it, we first need an auxiliary result.

Lemma 13. The type constructor Fin is injective: Fin $\mathrm{m} \equiv$ Fin $\mathrm{n}$ implies $\mathrm{m} \equiv \mathrm{n}$.

Proof. Given types A and B, a function $\mathrm{f}: \top \sqcup \mathrm{A} \rightarrow \top \sqcup \mathrm{B}$ induces a function $\mathrm{f}, \mathrm{:}$ : $\rightarrow$ B such that $f$, $a$ is $f$ a if it belongs to $B$, or $f$ tt otherwise. Using this construction, one can show that $\top \sqcup$ $\mathrm{A} \simeq \top \sqcup \mathrm{B}$ implies $\mathrm{A} \simeq \mathrm{B}$, and thus that $T \sqcup \mathrm{A} \equiv \top \sqcup \mathrm{B}$ implies $\mathrm{A} \equiv \mathrm{B}$ by univalence. We then conclude by induction using the fact that Fin (suc $\mathrm{n}$ ) $\equiv \top \sqcup$ Fin n. It is also possible to prove this fact without resorting to univalence [14].

Lemma 14. Given a type $\mathrm{A}$, if $\| \mathrm{A} \simeq$ Fin $\mathrm{m} \|$ and $\| \mathrm{A} \simeq$ Fin $\mathrm{n} \|$ then $\mathrm{m} \equiv \mathrm{n}$.

Proof. The equality between natural numbers is a proposition ( $\mathbb{N}$ is a set) and we can thus forget about the proposition truncations (by using the corresponding elimination rule). By transitivity, we should have Fin $\mathrm{m} \simeq$ Fin $\mathrm{n}$, thus Fin $\mathrm{m} \equiv$ Fin $\mathrm{n}$ by univalence and thus $\mathrm{m} \equiv \mathrm{n}$ by injectivity of the type constructor Fin by lemma 13.

Using this, one can deduce that being finite is a proper predicate:

Proposition 15. Being finite for a type is a proposition. 
Proof. Suppose given two proofs of is-finite A for some type A. Those have the same first component by lemma 14 and the same second component by definition of the propositional truncation. They are thus equal.

Remark 16. As a corollary of previous lemma, we get the fact that is-finite $\mathrm{A}$ is equivalent to $\| \Sigma \mathbb{N}$ $(\lambda \mathrm{n} \rightarrow \mathrm{A} \simeq$ Fin $\mathrm{n}) \|$, which could thus have served as an alternative definition.

Finite types satisfy the expected basic properties:

Proposition 17. The types $\perp$, $\top$ and $\mathrm{F}$ in $\mathrm{n}$ are finite. Finite types are closed under (dependent) sums and products.

Proposition 18. Given equivalent types $\mathrm{A}$ and $\mathrm{B}, \mathrm{A}$ is finite if and only if $\mathrm{B}$ is.

Proposition 19. Given types $\mathrm{A}$ and $\mathrm{B}$, we have that $\mathrm{A} \sqcup \mathrm{B}$ is finite if and only if both $\mathrm{A}$ and $\mathrm{B}$ are finite.

Proof. Suppose that A $\sqcup \mathrm{B}$ is finite. It can be shown that a decidable subtype of $\mathrm{F}$ in $\mathrm{n}$ is of the form Fin $k$. More precisely: given a family $P:$ Fin $n \rightarrow$ Type which is a predicate (i.e. $P$ i is a proposition for every $i$ in Fin $n$ ) and decidable (i.e. we have ( $P$ i) $\sqcup \neg\left(\begin{array}{l}P \\ i\end{array}\right)$ ), then there exists a natural number $\mathrm{k}$ such that $\Sigma$ (Fin $\mathrm{n}) \mathrm{P} \simeq$ Fin $\mathrm{k}$. By proposition 18 , we can deduce that a decidable subtype of a finite type is finite. Writing split : $A \sqcup B \rightarrow$ Bool for the function sending the elements of $A$ to true and the elements of $B$ to false, we have that $A$ is equivalent to the subtype of $A \sqcup B$ determined by the predicate $\mathrm{P}: \mathrm{A} \sqcup \mathrm{B} \rightarrow$ Type defined by $\mathrm{P} \mathrm{x}=$ split $\mathrm{x} \equiv$ true, which is decidable (because Bool has a decidable equality). We conclude that $A$ is finite, as a decidable subtype of $A \sqcup B$, which is supposed to be finite (and B is also finite for similar reasons).

Conversely, if $\mathrm{A}$ and $\mathrm{B}$ are finite, writing $\mathrm{m}$ and $\mathrm{n}$ for their cardinal, we have that $\mathrm{A} \simeq \mathrm{F}$ in $\mathrm{m}$ and $\mathrm{B} \simeq$ Fin $\mathrm{n}$, and thus $\mathrm{A} \sqcup \mathrm{B} \simeq$ Fin $\mathrm{m} \sqcup$ Fin $\mathrm{n} \simeq$ Fin $(\mathrm{m}+\mathrm{n})$ (we can ignore the propositional truncation because we are eliminating into the proposition of being finite).

Proposition 20. Finite types are sets.

Proof. Suppose given a finite type $(\mathrm{A},(\mathrm{n}, \mathrm{F}))$, where $\mathrm{A}$ is a type, $\mathrm{n}$ is a natural number and $\mathrm{F}$ a proof of $\| \mathrm{A} \simeq$ Fin $\mathrm{m} \|$. Being a set is a proposition [21, Lemma 3.3.5], by elimination of propositional truncation we can thus extract from $\mathrm{F}$ and equivalence $\mathrm{A} \simeq \mathrm{Fin} \mathrm{n}$, and we conclude by transporting the fact that Fin $\mathrm{n}$ is a set (it is easily shown to be decidable and thus a set by Hedberg's theorem [21, Theorem 7.2.5]).

We write FinType for the type of all finite types:

FinType : Type $_{1}$

FinType $=\Sigma$ Type is-finite

Note that this type is a large one, it lives in Type 1 . By proposition 15, two elements of this type are equal if and only if their first components (i.e. underlying types) are equal:

Lemma 21. Given elements $\mathrm{A}$ and $\mathrm{B}$ of FinType, we have $(\mathrm{A} \equiv \mathrm{B}) \simeq$ ( $f$ st $\mathrm{A} \equiv$ fst $\mathrm{B})$.

From this, we can deduce:

Proposition 22. FinType is a groupoid.

Proof. Given type finite types A and B, the type $\mathrm{A} \equiv \mathrm{B}$ is equivalent to the type of equalities between the underlying types of $A$ and $B$, which is a set by proposition 20 as the types of equalities between two sets. 


\subsection{A small axiomatization of the type finite types}

In this section, we show an important property: the type FinType of finite types is equivalent to a small type [4, Bij.agda]. This is a generalization of the following simple observation: every finite set is isomorphic to a set of the form $[n]=\{0, \ldots, n-1\}$ for some natural number $n$, so that the class of finite sets is equivalent to the set of natural numbers. However, FinType is a groupoid and not a set, in the sense of HoTT: there are non-trivial equalities between finite types which, by univalence, correspond to isomorphisms of finite types. For this reason, we do not expect that the type FinType is equivalent to the traditional type $\mathbb{N}$ of natural numbers, which has no non-trivial path between its element (it has decidable equality and thus is a set by Hedberg's theorem), but rather to a type that we call here $\mathbb{B}$, which has natural numbers as objects, but is moreover such that the group of path endomorphisms on an object $\mathrm{n}$ is the symmetric group on $\mathrm{n}$ elements. A more accurate picture of the situation than the equivalence between finite sets and natural numbers is thus the equivalence between the category Bij of finite sets an bijections and its skeleton, which has natural numbers as objects. Again, it is important to remark that Bij is not small (its collection of objects does not form a set) whereas its skeleton is.

The type $\mathbb{B}$ being constructed from constructors corresponding to the natural numbers, but also paths, it is natural to describe it as a higher inductive type [21, Section 6]. Those are not readily available in current plain version of Agda, but they can be simulated by working axiomatically with them, as done usually. The definition we give here is close to the one performed in [18] in order to define EilenbergMacLane spaces in homotopy type theory.

Definition 23. We axiomatize $\mathbb{B}$ as the small type such that

- it has natural numbers as objects:

obj : $\mathbb{N} \rightarrow \mathbb{B}$

- for every equivalence between finite sets there is a path in $\mathbb{B}$ between the corresponding natural numbers:

hom $:\{\mathrm{m} \mathrm{n}: \mathbb{N}\}(\alpha:$ Fin $\mathrm{m} \simeq$ Fin $\mathrm{n}) \rightarrow$ obj $\mathrm{m} \equiv$ obj $\mathrm{n}$

- the path associated to identity is the identity:

id-coh $:(\mathrm{n}: \mathbb{N}) \rightarrow \operatorname{hom}\{\mathrm{n}=\mathrm{n}\} \simeq$-refl $\equiv \operatorname{refl}$

- paths are compatible with composition:

comp-coh: $\{\mathrm{m} \mathrm{n} \circ: \mathbb{N}\}(\alpha:$ Fin $\mathrm{m} \simeq$ Fin $\mathrm{n})(\beta:$ Fin $\mathrm{n} \simeq$ Fin $\mathrm{o}) \rightarrow$ hom $(\simeq$-trans $\alpha \beta) \equiv$ hom $\alpha \cdot$ hom $\beta$

- there are no higher paths, i.e., $\mathbb{B}$ is a groupoid:

$\mathbb{B}$-is-groupoid : is-groupoid $\mathbb{B}$

We also need to postulate an appropriate elimination principle, which can be found in the formalization: it roughly states that, in order to define function of type $f: \mathbb{B} \rightarrow A$, for some groupoid $A$, it is enough to define:

- an element $f$ (obj $n$ ) of A for every natural number $n$,

- a path apd $f($ hom e) $: f(o b j m) \equiv f(o b j n)$ for every equivalence e : Fin $m \simeq$ Fin $\mathrm{n}$, 
in suitably coherent way. The slightly more readable non-dependent version of this elimination principle is

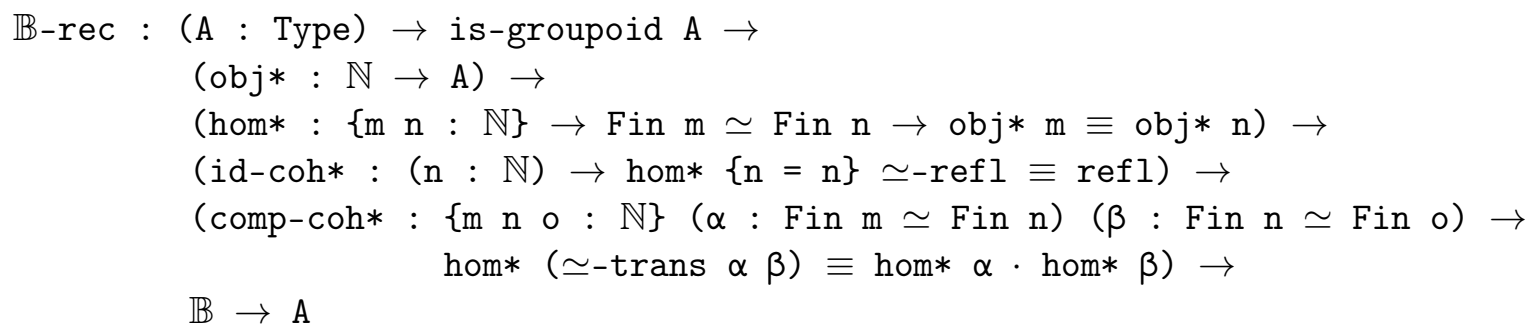

Finally, we also need to postulate two computation rules (implemented as rewriting rules). For simplicity we indicate the non-dependent versions here. Given a function $f: \mathbb{B} \rightarrow$ A obtained by applying $\mathbb{B}$-rec to some arguments with the above notations, we have

- $f(\operatorname{obj} n)=o b j * n$ for any natural number $n$,

- ap $f($ hom $\alpha)=$ hom* $\alpha$ for any equivalence $\alpha$ : Fin $m \simeq$ Fin $n$.

Remark 24. In the definition of hom, the reader might be surprised that we allow two different cardinalities of finite sets: given an equivalence $\mathrm{F}$ in $\mathrm{m} \simeq \mathrm{F}$ in $\mathrm{n}$, we necessarily have $\mathrm{m} \equiv \mathrm{n}$. But this "more general" definition simplifies the proofs in practice.

There is a canonical function $\mathbb{B}$-to-Fin : $\mathbb{B} \rightarrow$ Type which realizes the elements of $\mathbb{B}$ as finite types. More precisely, we define the function $\mathbb{B}$-to-FinType $: \mathbb{B} \rightarrow$ FinType by using the elimination principle $\mathbb{B}-r e c$ with the following arguments:

- A is the type FinType of finite types, which is a groupoid by proposition 22,

- obj* states that $\mathbb{B}$-to-FinType (obj $\mathrm{n}$ ) = Fin $\mathrm{n}$ (through the first computation rule),

- hom* states that, for an equivalence $\alpha$ : Fin $\mathrm{m} \simeq$ Fin $\mathrm{n}$, we have ap $\mathbb{B}$-to-FinType (hom $\alpha$ ) is the equality Fin $\mathrm{m} \equiv$ Fin $\mathrm{n}$ obtained from $\alpha$ by univalence (through the second computation rule).

The function $\mathbb{B}$-to-Fin can then be obtained by post-composing $\mathbb{B}$-to-FinType with the first projection, i.e. we forget about the proofs of finiteness.

One of the main contributions of this paper is the following theorem:

Theorem 25. The large type of finite types and the above type are equivalent: FinType $\simeq \mathbb{B}$.

Proof. The proof uses the "encode-decode method" introduced to compute the fundamental group of the circle [21, Section 8.1]. Given a natural number $\mathrm{n}$ and an element $\mathrm{b}$ of $\mathbb{B}$, we can encode the type of paths of type obj $n \equiv \mathrm{b}$ in $\mathbb{B}$ as the type Code $\mathrm{n} b$ defined by induction on $\mathrm{b}$. More precisely, we define the function Code $n: \mathbb{B} \rightarrow$ Set using $\mathbb{B}-r e c$ with the following arguments:

- A is the type Set of all sets, which is a groupoid,

- obj* states that Code $n$ (obj m) should be the type Fin $n \simeq$ Fin $m$ of equivalences between Fin $\mathrm{m}$ and $\mathrm{Fin} \mathrm{n}$,

- hom* $\alpha$ states, given an equivalence $\alpha$ : Fin $\mathrm{m} \simeq$ Fin $\mathrm{m}^{\prime}$, that ap (Code $\mathrm{n}$ ) $\alpha$ : (Fin $\mathrm{n} \simeq$ Fin $\mathrm{m}) \equiv$ (Fin $\mathrm{n} \simeq$ Fin $\mathrm{m}^{\prime}$ ) should be the equality between equivalences induced by postcomposition with $\alpha$. 
Then, we can define an "encoding" function e $:$ obj $\mathrm{n} \equiv \mathrm{b} \rightarrow$ Code $\mathrm{n}$ b such that e ref $\mathrm{l}=\simeq$-refl, and a "decoding" function $d:$ Code $\mathrm{n} \mathrm{b} \rightarrow$ obj $\mathrm{n} \equiv \mathrm{b}$ by a suitable induction on $\mathrm{b}$. It can be shown that $d \circ e$ is the identity and that, for $\alpha:$ Fin $m \simeq$ Fin $n$, we have e $(d($ hom $\alpha)) \equiv \alpha$. This can be used to deduce that the function ap $\mathbb{B}$-to-FinType : obj $\mathrm{m} \equiv$ obj $\mathrm{n} \rightarrow$ Fin $\mathrm{m} \equiv$ Fin $\mathrm{n}$ is an equivalence, a thus that the function $\mathbb{B}$-to-FinType is an embedding (by induction). The function $\mathbb{B}$-to-FinType is easily shown to be surjective: any finite type $A$ is equal to $\mathbb{B}$-to-FinType (obj $n$ ) where $\mathrm{n}$ is the cardinality of $\mathrm{A}$. We finally deduce that the function is an equivalence since it is both an embedding and surjective, see [21, Theorem 4.6.3].

\section{A cartesian closed bicategory of finitary polynomials}

\subsection{Finitary polynomials}

Thanks to the notion of finiteness for types, we define in [4, FinPolynomial . agda] finitary polynomials following the explanations of section 2.3.

Definition 26. A polynomial is finitary when, for each operation c, the total space of its parameters is finite:

is-finitary $:(P: I \rightsquigarrow J) \rightarrow$ Type

is-finitary $P=\{j: J\}(c: O p P j) \rightarrow$ is-finite $(\Sigma I(\lambda i \rightarrow P m P$ i $c)$ )

Remark 27. Another definition of being finitary could be to require that each space of parameters is finite:

is-finitary' $P=(i: I)\{j: J\}$ ( $c: O p P j$ ) $\rightarrow$ is-finite (Pm $P$ i $c$ )

but one quickly convinces oneself that this notion is not suitable: identity polynomials are not generally finitary in this sense, and being finitary is not stable under composition.

Proposition 28. The cartesian bicategory of theorem 7 restricts to a bicategory whose morphisms are finitary polynomials.

Proof. Finitary polynomials can be shown to be stable under the required operations (composition, product) using the closure of finite type under the operations of proposition 17.

By using similar arguments as in section 6.2, the proof of section 4.2 can be refined to show that, ignoring size issues, the cartesian bicategory of finitary polynomials in groupoids admits Exp $I \times J$ as internal hom, where Exp is now defined as

Exp : Type $\rightarrow$ Type $_{1}$

$\operatorname{Exp} I=\Sigma(I \rightarrow$ Type $)(\lambda F \rightarrow$ is-finite $(\Sigma I F))$

i.e., we restrict to families whose total space is finite. Moreover, through the equivalence between families and slices (see theorem 10), it can be shown that the above type is equivalent to the one of finite sets over I:

Lemma 29. The above definition of the exponential is equivalent to the following one:

Exp : Type $\rightarrow$ Type $_{1}$

$\operatorname{Exp} \mathrm{I}=\Sigma$ FinType $(\lambda \mathrm{N} \rightarrow$ fst $\mathrm{N} \rightarrow \mathrm{I})$

In turn, by theorem 25 , we have that this type is equivalent to the one of elements of $\mathbb{B}$ over $I$ :

Lemma 30. The above definition of the exponential is equivalent to the following one:

Exp : Type $\rightarrow$ Type

$\operatorname{Exp} I=\Sigma \mathbb{B}(\lambda \mathrm{b} \rightarrow \mathbb{B}$-to-Fin $b \rightarrow I)$

This is now small type, and thus a reasonable candidate for the right definition of the exponential. 


\subsection{A cartesian closed bicategory}

We can finally show that the cartesian bicategory of finitary polynomials (proposition 28) is closed. Before doing so, we first prove a useful lemma:

Lemma 31. Given types $\mathrm{I}$ and $\mathrm{J}$ and a family $\mathrm{A}:(\mathrm{I} \sqcup \mathrm{J}) \rightarrow$ Type, we have that the total type $\Sigma(\mathrm{I} \sqcup \mathrm{J}) \mathrm{A}$ is finite if and only if both types $\Sigma \mathrm{I}(\mathrm{A} \circ \mathrm{inl})$ and $\Sigma \mathrm{J}(\mathrm{A} \circ$ inr $)$ are finite.

Proof. We have that $\Sigma(I \sqcup \mathrm{J})$ A is equivalent to $(\Sigma \mathrm{I}(\mathrm{A} \circ$ inl $)) \sqcup(\Sigma \mathrm{J}(\mathrm{A} \circ$ inr $))$ and we conclude by proposition 18 and proposition 19 .

As suggested in previous section, we define the exponential as

Exp : Type $\rightarrow$ Type

$\operatorname{Exp} I=\Sigma \mathbb{B}(\lambda \mathrm{b} \rightarrow \mathbb{B}$-to-Fin $b \rightarrow I)$

and finally show our main theorem, by adapting the naive constructions of section 4.2 [4, FinPolynomial . agda, CurryUncurry . agda, UncurryCurry . agda]:

Theorem 32. The cartesian bicategory of finitary polynomials is closed, with $\operatorname{Exp} \mathrm{I} \times \mathrm{J}$ as internal hom from I to J.

Proof. We can define a function

curry : $((\mathrm{I} \sqcup \mathrm{J}) \rightsquigarrow \mathrm{K}) \rightarrow(\mathrm{I} \rightsquigarrow(\operatorname{Exp} \mathrm{J} \times \mathrm{K}))$

essentially as in section 4.2 except that we have to turn the output $j \mathrm{j}: \mathrm{J} \rightarrow$ Type of the currying of an operation c into an element of $\operatorname{Exp} \mathrm{J}$. By lemma 31, $\mathrm{j} j$ is a finite type, and thus induces an element of $\mathbb{B}$ by applying the canonical function card : FinType $\rightarrow \mathbb{B}$ given theorem 25 which can serve as first component in $\operatorname{Exp} \mathrm{J}$, and the second component can be deduced from the proof that card is an inverse to $\mathbb{B}$-to-FinType. The fact that the resulting polynomial is finite follows from lemma 31. Similarly, we can define a function

uncurry : $(\mathrm{I} \rightsquigarrow(\operatorname{Exp} \mathrm{J} \times \mathrm{K})) \rightarrow((\mathrm{I} \sqcup \mathrm{J}) \rightsquigarrow \mathrm{K})$

which is again given as in section 4.2, using theorem 25 to use elements of the exponential and lemma 31 to prove the required finiteness of types. The two functions can be shown to be mutually inverse. Finally, the bijection should be checked to be natural (this last part is not fully formalized yet).

\section{Future work}

In this work, we have constructed a cartesian closed bicategory of finitary polynomial functors in groupoids, in the setting of type theory. A natural next step is to translate these constructions in to the traditional set-theoretic setting, in order to ease the comparison with more traditional approaches to polynomial functors. As mentioned in section 2.4, we do not expect this task to be particularly easy.

A second aspect in which we wish to push investigations on this bicategory is its relation with linear logic. Namely, the bicategory of spans in groupoids can be understood as the full subbicategory of polynomials of the form (1) where the morphism $p$ is the identity. This subbicategory is monoidal with the tensor induced on objects by cartesian product of groupoids. The inclusion functor admits a left adjoint which is a strong lax monoidal functor. This allows to model the exponential of linear logic [19] and will be detailed elsewhere. We also expect that the constructions of differential linear logic can be interpreted in the model. 
Finally, our model is close to the one of generalized species, which is also a model of (differential) linear logic [5, 7] inspired by combinatorial species [13]. We would like to understand the relationship between those two models, which is hinted at in [16, Section 3.9]: one of the main difference is that whereas generalized species are be composed using traditional composition of profunctors, which involves a quotient, homotopy polynomial functors perform a homotopy quotient, and we should be able to obtain the first from the second by suitably discarding homotopical information (i.e., "taking $\pi_{0}$ ").

On the long term, we finally want to investigate the generalization to polynomials in $\infty$-groupoids, as first studied in [10]. This would make more transparent the comparison with the type theoretic formalization, but we expect the study of this model to be much more involved on a technical level. The definitions given here should work identically without the hypothesis that types are groupoids, and the resulting polynomials should organize as an $\infty$-category instead of a bicategory, but there is currently no known definition of $\infty$-categories inside type theory, preventing us from formally showing this result for now.

\section{References}

[1] Benedikt Ahrens, Dan Frumin, Marco Maggesi \& Niels van der Weide (2019): Bicategories in Univalent Foundations. In: 4th International Conference on Formal Structures for Computation and Deduction, pp. 1-17, doi:10.4230/LIPIcs.FSCD.2019.5.

[2] Thorsten Altenkirch, Neil Ghani, Peter Hancock, Conor McBride \& Peter Morris (2015): Indexed containers. Journal of Functional Programming 25, doi:10.1017/S095679681500009X.

[3] Nathanael Arkor \& Marcelo Fiore (2020): Algebraic models of simple type theories: A polynomial approach. In: Proceedings of the 35th Annual ACM/IEEE Symposium on Logic in Computer Science, pp. 88-101, doi:10.1145/3373718.3394771.

[4] Eric Finster, Maxime Lucas, Samuel Mimram \& Thomas Seiller (2021): https://github.com/smimram/fibred-polynomials.

[5] Marcelo Fiore (2004): Generalised species of structures: Cartesian closed and differential structure.

[6] Marcelo Fiore (2014): Analytic functors between presheaf categories over groupoids. Theoretical Computer Science 546, pp. 120-131, doi:10.1016/j.tcs.2014.03.004.

[7] Marcelo Fiore, Nicola Gambino, Martin Hyland \& Glynn Winskel (2008): The cartesian closed bicategory of generalised species of structures. Journal of the London Mathematical Society 77(1), pp. 203-220, doi:10.1112/jlms/jdm096.

[8] Marcelo P Fiore (2007): Differential structure in models of multiplicative biadditive intuitionistic linear logic. In: International Conference on Typed Lambda Calculi and Applications, Springer, pp. 163-177, doi:10.1007/978-3-540-73228-0_13.

[9] Nicola Gambino \& Joachim Kock (2013): Polynomial functors and polynomial monads. In: Mathematical Proceedings of the Cambridge Philosophical Society, 1, Cambridge University Press, pp. 153-192, doi:10.1017/S0305004112000394.

[10] David Gepner, Rune Haugseng \& Joachim Kock (2017): $\infty$-Operads as Analytic Monads. arXiv preprint arXiv:1712.06469.

[11] Jean-Yves Girard (1988): Normal functors, power series and $\lambda$-calculus. Annals of pure and applied logic 37(2), pp. 129-177, doi:10.1016/0168-0072(88)90025-5.

[12] Ryu Hasegawa (2002): Two applications of analytic functors. Theoretical Computer Science 272(1-2), pp. 113-175, doi:10.1016/S0304-3975(00)00349-2.

[13] André Joyal (1986): Foncteurs analytiques et especes de structures. In: Combinatoire énumérative, Springer, pp. 126-159, doi:10.1007/BFb0072514. 
[14] Donnacha Oisín Kidney (2019): A Small Proof that Fin is Injective. https://doisinkidney.com/posts/2019-11-15-small-proof-fin-inj.html.

[15] Joachim Kock (2009): Notes on polynomial functors. Manuscript, version.

[16] Joachim Kock (2012): Data types with symmetries and polynomial functors over groupoids. Electronic Notes in Theoretical Computer Science 286, pp. 351-365, doi:10.1016/j.entcs.2013.01.001.

[17] Joachim Kock, André Joyal, Michael Batanin \& Jean-François Mascari (2010): Polynomial functors and opetopes. Advances in Mathematics 224(6), pp. 2690-2737, doi:10.1016/j.aim.2010.02.012.

[18] Daniel R Licata \& Eric Finster (2014): Eilenberg-MacLane spaces in homotopy type theory. In: Proceedings of the Joint Meeting of the Twenty-Third EACSL Annual Conference on Computer Science Logic (CSL) and the Twenty-Ninth Annual ACM/IEEE Symposium on Logic in Computer Science (LICS), pp. 1-9, doi:10.1145/2603088.2603153.

[19] Paul-André Melliès (2009): Categorical semantics of linear logic. Panoramas et syntheses 27, pp. 15-215.

[20] Paul Taylor (1989): Quantitative domains, groupoids and linear logic. In: Category Theory and Computer Science, Springer, pp. 155-181, doi:10.1007/BFb0018351.

[21] The Univalent Foundations Program (2013): Homotopy Type Theory: Univalent Foundations of Mathematics. https://homotopytypetheory.org/book, Institute for Advanced Study.

[22] Jakob Vidmar (2018): Polynomial functors and W-types for groupoids. Ph.D. thesis, University of Leeds.

[23] Mark Weber (2015): Polynomials in categories with pullbacks. Theory and applications of categories 30(16), pp. 533-598. 